\title{
Théologiques
}

Théologiques

\section{Derniers repas}

\section{Olivier Bauer}

Volume 23, numéro 1, 2015

\section{Derniers repas}

URI : https://id.erudit.org/iderudit/1040863ar

DOI : https://doi.org/10.7202/1040863ar

Aller au sommaire du numéro

\section{Éditeur(s)}

Faculté de théologie et de sciences des religions, Université de Montréal

ISSN

1188-7109 (imprimé)

1492-1413 (numérique)

Découvrir la revue

Citer ce document

Bauer, O. (2015). Derniers repas. Théologiques, 23(1), 7-13.

https://doi.org/10.7202/1040863ar

Ce document est protégé par la loi sur le droit d'auteur. L'utilisation des services d'Érudit (y compris la reproduction) est assujettie à sa politique d'utilisation que vous pouvez consulter en ligne.

https://apropos.erudit.org/fr/usagers/politique-dutilisation/
Cet article est diffusé et préservé par Érudit.

Érudit est un consortium interuniversitaire sans but lucratif composé de l’Université de Montréal, l'Université Laval et l'Université du Québec à Montréal. Il a pour mission la promotion et la valorisation de la recherche. https://www.erudit.org/fr/ 


\title{
Derniers repas
}

\author{
Olivier BAUER" \\ Théologie pratique \\ Université de Lausanne
}

\section{Qu'aimeriez-vous manger pour votre dernier repas?}

La question peut paraître saugrenue ou incongrue. Après tout, sauf à de très rares exceptions, personne n'a la chance de planifier son dernier repas. Le plus souvent, la mort survient de manière inattendue. Et la plupart d'entre nous risque bien de n'avoir, en guise de dernier repas, qu'une injection de solution sucrée ou salée. Cette froide réalité n'empêche pourtant pas d'imaginer le menu de son dernier repas, d'un repas ultime, d'un repas qui pourrait résumer une existence, dire ce que l'on a été ou ce que l'on aurait voulu être.

Parmi tout ce qui lie alimentation et spiritualité, le thème du dernier repas me paraît particulièrement significatif. Sa proximité avec la mort, qu'elle soit réelle ou symbolique, correspondant au dénouement d'une existence, lui confère une valeur unique. C'est sans doute ce qui explique qu'il apparaisse sous des formes et dans des lieux variés, si variés qu'il m'est évidemment impossible de prétendre tous les identifier. Mais ils partagent pourtant des traits communs, des similitudes ou des ressemblances qui me permettent de les organiser. Car des grandes tendances se dégagent:

* Olivier Bauer est professeur ordinaire à la Faculté de théologie et de sciences des religions à l'Université de Lausanne. Il est directeur de l'Institut lémanique de théologie pratique. Ses recherches portent notamment sur le rôle des six sens dans la transmission de la foi (ritualités, éducation de la foi), sur le sport et la religion (sport comme religion, aumônerie des milieux sportifs), ainsi que sur l'alimentation et la spiritualité (impact des religions sur les habitudes alimentaires, spiritualisation de l'alimentation). Il rédige actuellement un ouvrage d'érudition sur les aliments figurant sur les images médiévales de la Cène. Il a récemment publié (2017): Les cultes des protestant.e.s. Méthodes originales pour approcher les rites, Genève, Labor et Fides. 
certains derniers repas sont des repas consommés, d'autres des repas représentés; certains derniers repas sont réellement les derniers, d'autres le sont symboliquement. En combinant les quatre catégories, j'identifie quatre types de dernier repas.

1. Des repas représentés qui sont véritablement les derniers.

2. Des repas représentés qui sont symboliquement les derniers.

3. Des repas consommés qui sont symboliquement les derniers.

4. Des repas consommés qui sont véritablement les derniers.

On verra que ce volume de Théologiques parcourt tous ces types, des types que quelques exemples permettront d'illustrer.

Le repas que le Bouddha prend au moment de sa mort appartient aux «repas représentés qui sont véritablement les derniers »; il y aurait mangé du sūkara-maddava, un hapax qui pourrait indiquer un plat à base de porc (Wasson 1982); ce qui ne serait pas anodin, car la consommation de viande par un tel personnage à un tel moment pourrait remettre en cause un bouddhisme végétarien. Y appartient aussi le repas que les quatre évangiles font prendre à Jésus à la veille de sa crucifixion; trois d'entre eux le font partager du pain et du fruit de la vigne qu'il identifie à son corps et à son sang, tandis que le quatrième le présente lavant les pieds de ses disciples, ce qui n'est pas plus anodin: les Églises chrétiennes ont fait du premier geste un rite, le christianisme et les cultures qu'il sous-tend ont survalorisé le pain et le vin. Mais les derniers repas représentés existent aussi sous une forme atténuée, celle des «repas représentés qui sont symboliquement les derniers ", des derniers repas avant quelque chose d'autre. Raconté dans la Bible juive, l'un de ces repas fera date, celui où Dieu ordonne au peuple d'Israël de consommer un jeune agneau et des pains sans levains les sandales au pied, prêt à fuir l'Égypte. Ces quelques exemples pourraient faire penser que le motif du dernier repas est un motif exclusivement religieux. Or, ce n'est pas le cas. L'importance que les religions donnent au dernier repas témoigne seulement de la place qu'il occupe dans l'imaginaire en même temps qu'il la renforce. Les artistes s'en sont emparé et l'ont largement utilisé, sous les deux formes des "repas représentés qui sont véritablement les derniers" et "des repas représentés qui sont symboliquement les derniers ». Certains derniers repas réinterprètent directement un motif religieux comme en témoignent les innombrables représentations picturales de la Cène et, dans une mise en abyme infinie, depuis que Leonardo da Vinci a peint son Cenacolo (da Vinci 1494), les 
représentations de ces représentations. Mais les artistes créent souvent leurs propres derniers repas. Dans le Festin de Babette, Karen Blixen (2007) décrit un repas français, ultime occasion de faire l'expérience de la grâce et dans La Panne, Friedrich Dürrenmatt raconte un repas où les convives se transforment en juge et finissent par prononcer une condamnation à mort (Dürrenmatt 1956); avec La Grande Bouffe, Marco Ferreri réalise un repas où les convives mangent jusqu'à en mourir (Ferreri 2006); Jacques Brel chante À mon dernier repas, un repas qui réunit tout ce qu'il a aimé et tous ceux qu'il a aimés (Brel 1964) et dans Le chat du café des artistes, Jean-Pierre Ferland chante un repas où c'est son cadavre qui est jeté pour être mangé (Ferland 1970); enfin Melanie Dunea demande à des grands chefs le menu de leur dernier repas, ce qui lui permet d'en tirer deux livres (Dunea 2007; 2011).

Le dernier repas consommé existe d'abord sous la forme du "repas consommé qui est symboliquement le dernier». Chaque année, des juifs partagent le repas du Seder pour se souvenir que Dieu les a délivrés de l'esclavage en Égypte; chaque dimanche, les Églises chrétiennes célèbrent des eucharisties ou des cènes pour réitérer la mort et la résurrection de Jésus Christ ou pour en faire mémoire; les catholiques prennent à Mardi-Gras un dernier "vrai repas » avant le début du Carême pascal; pendant le mois du Ramadan, les musulmans prennent, chaque matin à l'aube, As-Sahûr, leur dernier repas avant de jeûner tout au long de la journée. Mais les religions n'ont pas non plus l'apanage ou le monopole de ce type de dernier repas. Des gens ritualisent leur dernier repas avant un moment charnière de leur existence: un départ à l'étranger ou un retour à la maison; une nouvelle étape dans leur existence, un enterrement de vie de garçon ou de jeune fille, un pot de départ à la retraite. Les « repas consommés qui sont véritablement les derniers » sont plus rares. On trouve en catholicisme le viatique, cette ultime communion, cette ultime hostie que le prêtre donne au mourant; on trouve dans les religions traditionnelles, particulièrement en Afrique et en Asie, les repas, les nourritures et les libations partagées avec les morts au cours des funérailles, on trouve encore, depuis longtemps, les derniers repas des condamnés à mort; on trouve aussi, de plus en plus publiquement, les derniers repas des candidats au suicide, au suicide assisté ou à l'euthanasie. Mais on trouve aussi ceux que l'on organise à l'occasion d'un décès, ceux qui consolent les survivants; on trouve en judaïsme le repas de consolation; fourni par les proches, il suit l'inhumation (Hidiroglou 2013); on trouve enfin, dans les Alpes suisses, l'ancienne coutume de mettre de côté un formage et du vin pour ses propres funérailles (Preiswerk 1983). 
Ce dossier de Théologiques explore donc le motif du dernier repas sous ses diverses formes. Il l'explore, au sens où il ne prétend pas épuiser le sujet, mais ouvrir une réflexion et proposer des pistes pour la mener. On pensera peut-être, c'est du moins ce que j'espère, que chaque article pourrait, et même devrait, être accompagné d'autres articles qui porteraient sur des thématiques identiques ou similaires. Mais si chaque article fait naître un tel sentiment, c'est qu'il signale et travaille une thématique particulière, qu'il donne envie d'en savoir plus, de savoir comment on pourrait traiter mêmement d'autres derniers repas, comment on pourrait traiter autrement les mêmes derniers repas. Et c'est tout le mérite des cinq auteurs de lancer la réflexion sur un sujet encore très peu travaillé, surtout de manière transversale, multidisciplinaire et internationale, d'offrir des résultats d'une telle recherche et de proposer des méthodes pour la poursuivre. J'espère qu'on leur rendra justice.

À titre de directeur du volume, il me reste encore à souligner les principaux apports de chacun des auteurs. Tâche plaisante, mais difficile et surtout pleine d'orgueil.

Les deux premiers articles du volume analysent deux représentations du même dernier repas.

Le volume s'ouvre avec mon analyse littéraire des récits bibliques de la Cène. Une telle entrée en matière pourrait paraître normale, voire convenue, dans le cadre d'une thématique sur les derniers repas. Sauf que je montre que, contrairement à l'idée largement répandue, la Cène n'est pas le dernier repas de Jésus. Ressuscité, il donne encore à manger et même mort, il mange encore: du pain sur la route d'Emmaüs, du poisson, peutêtre du miel au bord du lac de Tibériade. Ce pourrait être seulement anecdotique, mais les enjeux ecclésiologiques ne sont pas négligeables. Car on peut lire dans les récits de ces repas post mortem, l'indication que le mouvement de Jésus et le christianisme primitif faisaient de tous les repas et d'aliments très divers des actes de foi. L'analyse de la première lettre de Paul aux Corinthiens ainsi que la lecture des Actes des Apôtres et de la Didachê le confirme: "parce que la Cène n'est que l'avant-dernier repas de Jésus, l'eucharistie n'est que l'un des repas où il est possible de rencontrer le Ressuscité ». C'est seulement au milieu du II $^{\mathrm{e}}$ siècle que l'Église chrétienne confiera cette fonction à la seule eucharistie.

Dans le deuxième article, comme en écho logique à cette lecture des récits de la Cène, Nancy Labonté et moi-même proposons notre analyse de la réinterprétation qu'en a faite Leonardo da Vinci. Avec nos bagages de sémioticienne pour l'une et de théologien pour l'autre, nous nous 
aventurons dans l'histoire de l'art. L'identification des aliments par une observation méticuleuse du tableau nous conduit à émettre des hypothèses sur les raisons qui ont pu pousser le peintre milanais à les faire figurer sur la table de son Cenacolo. Aurait-il choisi les aliments que mentionnent les évangiles, ceux du repas du Seder ou plutôt ceux qu'il aimait manger? Les aurait-il privilégiés pour leur valeur symbolique en christianisme ou pour inscrire sa propre théologie dans la fresque qu'il peignait dans le réfectoire d'un couvent? La présence d'anguille, la valeur des oranges, la position de la salière nous font privilégier l'hypothèse d'une réinterprétation personnelle et subversive de la Cène de Jésus. Elle serait «un jeu de dupe, une peinture en trompe-l'œil, un repas en "trompe-la-bouche" ".

Ce n'est certainement pas par hasard que j'ai placé l'article de Muriel Schmid au centre de ce volume. Son article nous fait radicalement changer de registre, puisque l'auteure y analyse un dernier repas consommé, qui est, pour celles et ceux qui le mangent, véritablement et définitivement le dernier. Forte d'une double expertise en théologie et en études pénitentiaires, Muriel Schmid démystifie les derniers repas offerts aux condamnés à mort dans les prisons étatsuniennes. Elle réinscrit cette coutume dans le contexte de l'histoire de la prison et montre comment les changements de contexte en modifient la valeur. À «l'époque puritaine ", c'est-à-dire à la fin $\mathrm{du} \mathrm{XVII}^{\mathrm{e}}$ siècle et au début du XVIII ${ }^{\mathrm{e}}$ siècle, quand la condamnation à mort était un lieu possible de conversion et de salut, le dernier repas offert aux condamnés avant leur exécution pouvait être une réitération de la Cène de Jésus, qui est, rappelle Muriel Schmid, «le repas d'un condamné qui devait bientôt être exécuté ». Mais quand, dès la moitié du XIX ${ }^{\mathrm{e}}$ siècle, la peine de mort devient le moyen d'éliminer définitivement des criminels perçus comme des monstres, le dernier repas offert aux condamnés à mort change de fonction. Il pourrait servir à réconforter celui ou celle qui va être exécuté. Mais les restrictions et les limites imposées aux choix des prisonniers prouvent qu'il est devenu une manière pour la société d'affirmer qu'elle reste «humaine» même dans les pires exécutions.

Les deux derniers articles du volume s'intéressent à ce qui suit un dernier repas symbolique. Ils viennent ainsi relativiser l'importance ou la centralité du motif du dernier repas. Ce qui me réjouit. Heureux le directeur d'un volume qui a la chance d'apprendre encore!

Fanny Parise s'intéresse au changement de régime alimentaire et à la manière dont il est mis en scène. Par une "approche ethnographique et netnographique», elle montre que les transitions valorisent le premier 
repas plutôt que le dernier: premier repas végétarien, premier repas végane, premier repas liquide, etc. Ce qui se comprend aisément, puisque ce premier «nouveau repas» a comme effet immédiat de dévaloriser le dernier "ancien repas» et tous ceux qui l'ont précédé. Mais elle montre aussi que ce premier repas peut arriver par hasard, qu'il peut se répéter, que finalement, il n'advient comme premier repas que dans une «narration a posteriori », quand les «transitionneurs» le racontent et l'illustrent. Elle montre enfin que la mise en scène des premiers repas sur les réseaux sociaux donne ou redonne la parole aux consommateurs. Ils peuvent ainsi témoigner de leur nouvelle pratique nutritionnelle qui se révèle être un nouveau système de croyances. Car la consommation est un lieu d'expérience spirituelle: on y charge de pouvoir ou de mana aussi bien les aliments que l'on consomme que les objets qui permettent de les produire.

Mais qu'en est-il quand le dernier repas n'est suivi d'aucun autre repas? C'est pour répondre à cette interrogation que Florence Ollivry examine le sens et la valeur du jeûne. Au travers d'un parcours exhaustif en judaïsme, en christianisme et en islam, tout en nuances et en différences, elle prouve que «l'absence d'appétit pour la nourriture» ne signifie pas forcément « «l'absence d'appétit pour la vie». Parce qu'il pourrait relever d'un désir morbide, les trois monothéismes prennent soin de réguler le jeûne, de lui donner du sens. Le judaïsme lui donne une valeur morale et spirituelle: il incarne la soumission à Dieu, il sanctifie la vie. Le christianisme, considérant que le fait même de manger présente des risques, prône le jeûne comme un moyen de les éviter: le christianisme oriental pratique alors un jeûne de pénitence ou de purification, tandis que le christianisme occidental ou le catholicisme romain pratique le jeûne pour maîtriser les passions. L'islam, quant à lui, assimile le jeûne à «l'abandon d'un acte» pour que l'être humain se délivre de ses penchants égoïstes et qu'il signifie la gratitude à l'égard de Dieu.

Pour terminer ce liminaire, je tiens à souligner que ce volume rassemble les articles de quatre auteures et d'un seul auteur. Même si cette composition reste quelque peu aléatoire, les auteur.e.s sollicité.e.s restant toujours libres d'accepter ou de refuser de contribuer puis de remettre ou non leur article, je ne crois pas que cette surreprésentation féminine soit totalement le fait du hasard. Elle témoigne sans doute d'une attention portée aux sujets plus ordinaires, à la valeur spirituelle ou symbolique d'aspects de la vie quotidienne que certains jugeront, à tort, triviaux. Cette attention n'est pas forcément féminine, mon propre texte suffirait à réfuter une telle hypothèse. Elle relève probablement d'abord d'intérêts personnels 
plus que du genre. Mais en l'occurrence, ce sont des chercheuses qui ont accepté d'écrire sur le thème du dernier repas et qui ont pris du temps pour le faire. Le résultat me réjouit et je les en remercie.

Et vous, qu'aimeriez-vous manger pour votre dernier repas? J'espère que la lecture de ce dossier de Théologiques vous permettra de renouveler votre manière de répondre à la question. Bonne lecture! Et bon appétit!

\section{Références}

Blixen, K. (2007), «Le festin de Babette», Les contes, Paris, Gallimard (Quarto).

BREL, J. (1964), Le dernier repas (Vol. Infiniment), Universal Music Division Barclay.

Dunea, M. (2007), My Last Supper. 50 Great Chefs and Their Final Meals. Portraits, Interviews, and Recipes ( $1^{\text {st }}$ U.S.), New York, Bloomsbury.

Dunea, M. (2011), My Last Supper. The Next Course. 50 Great Chefs and Their Final Meals. Portraits, Interviews, and Recipes, Emmaus/New York, Rodale.

Dürrenmatt, F. (1956), Die Panne; eine noch mögliche Geschichte, Zürich, Verlag der Arche.

Ferland, J.-P. (1970), Le chat du Café des artistes (Vol. Jaune), Barclay.

Ferreri, M. (2006), La grande bouffe, Paris, Roissy Films.

Hidiroglou, P. (2013), «Nourritures des vivants, mémoire des morts dans les sociétés juives ", Ethnologie française, 43/4, p. 623-632.

Preiswerk, Y. (1983), Le Repas de la Mort. Catholiques et protestants aux enterrements. Visages de la culture populaire en Anniviers et aux Ormonts, Sierre, Monographic.

VINCI, L. da (1494), Ultima Cena, $460 \times 880 \mathrm{~cm}$, peinture murale à la détrempe, Couvent dominicain de Santa Maria delle Grazie.

Wasson, R. G. (1982), "The Last Meal of the Buddha ", Journal of the American Oriental Society, 102/4, p. 591-603. 